Forschende

Komplementärmedizin und

Klassische Naturheilkunde
Editorial

Forsch Komplementärmed Klass Naturheilkd 2001;8:333-334

\title{
Die Bedeutung des Begriffs «normal»
}

Der Begriff «normal» spielt bei ärztlichen Handlungen eine wesentliche Rolle. Dies betrifft in erster Linie die Diagnostik, hat aber dann naturgemäss auch Auswirkungen auf die Therapie. Bei etwas näherer Betrachtung stellt sich jedoch heraus, dass eine Definition dieses Begriffs, die auch praktisch eingesetzt werden kann, gar nicht so einfach ist. Häufig beschreibt «normal» etwas, was als typisch, üblich oder natürlich empfunden wird. So entspricht etwa eine normale Körpertemperatur jener Kerntemperatur, die üblicherweise bei gesunden Menschen gemessen werden kann und ein «normales» Verhalten beschreibt etwas, was üblicherweise gesellschaftlich erwartet und akzeptiert wird. In der Medizin wird der Begriff «normal» bekanntlich auch dazu verwendet, um Gesundheit von Krankheit zu differenzieren. Dies hat sich in der klinischen Medizin bei eindeutigen und klinisch manifesten Krankheiten zweifelsohne bewährt, es ist jedoch diskussionswürdig, ob das Konzept einer Krankheitsdiagnose, welches auf der Abweichung vom Normalen basiert, auch bei jenen Leidenszuständen praktikabel ist, die häufig in der Komplementärmedizin Ursache der Inanspruchnahme ärztlicher Hilfe sind.

Doch auch im Bereich der klinischen Medizin ist der Begriff «normal» nicht eindeutig definiert. So kann «normal» typisch bedeuten, wenn eine allgemein akzeptierte Praxis die Bezugsgrundlage ist. «Normal» kann aber auch durchschnittlich meinen, wenn das beschrieben werden soll, was am repräsentativsten in einer Gruppe ist. Wenn es hingegen um die Sicherheit in einer klinischen Studie geht, so kann «normal» unschädlich oder harmlos bedeuten. In der Genetik andererseits definiert dieser Begriff das, was für das Überleben am besten geeignet ist. «Normal» kann sich aber auch auf einen definierten Standard beziehen, wie das z.B. für einen «normalen» Blutdruck zutrifft. Eine «normale» Immunitätslage hingegen beschreibt einen natürlicherweise vorkommenden Zustand. Ein «normaler» Untersuchungsbefund wird als Freisein von Krankheiten aufgefasst, eine «normale» Ernährungsform steht oft für eine ausgewogene Ernährung. In allen diesen Fällen, wird der Begriff «normal» dazu verwendet, um einen üblichen Befund oder einen erwarteten Zustand zu definieren. In der Medizin besteht jedoch die Gefahr, dass der Begriff «normal» dazu verwendet wird um etwas zu beschreiben, was als perfekt oder ideal empfunden wird. Diese Verwendung des Begriffs stammt vom Konzept der klinischen Norm, die als fixierter oder idealer Standard definiert ist. Es wird dabei also irgendein aktuell erhobener Messwert mit dem idealen Standard verglichen. Die Definition einer normalen PlasmacholesterinKonzentration repräsentiert z.B. einen idealen Standard und nicht den Durchschnittswert, der in der erwachsenen Bevölkerung gefunden wird. Die Medizin hat viele klinische Normen für diverse Parameter definiert, indem man mathematisch Werte aus Resultaten klinischer Tests ableitete und dann entschieden wurde, welcher dieser Werte in einem Normalbereich liegt. Dabei wird dieser Normalbereich auf verschiedene Art und Weise bestimmt, z.B. durch die Berechnung eines Durchschnitts, durch eine empirische Entscheidung darüber, was gesund ist, oder durch Abschätzen dessen, was ein optimaler Zustand sein sollte. Bei allen diesen Definitionen besteht jedoch die Gefahr Normalwerte als Kennzeichen des Idealen, Perfekten oder ausserordentlich Gesunden aufzufassen. In diesem Fall repräsentiert der Begriff «normal» nicht mehr das, was üblich, typisch oder allgemein ist, sondern das was erwünscht ist. Eine solche Definition der Norm als das Ideale oder Perfekte, kann beträchtliche Probleme hervorrufen. Perfektion ist schon theoretisch schwer möglich, in der Praxis aber wohl undenkbar. Eine perfekte Gesundheit über die gesamte Lebensspanne ist unerreichbar. Gesundheitsbeeinträchtigung und Krankheit sind ein normaler Teil des Lebens. Die fortwährende Jagd nach der perfekten Gesundheit und die gleichzeitige Unmöglichkeit, diese zu erreichen, kann auch dazu führen, jene zu diskriminieren, die ohnehin die Last einer Krankheit zu tragen haben.

Die Auffassung, dass Abweichungen vom Idealzustand als abnormal und damit auch als krankhaft zu gelten haben, kann auch deshalb problematisch sein, weil solche Abweichungen von einem Idealzustand mitunter keinerlei klinische Bedeutung oder Konsequenz aufweisen.

Impliziert in die Idee einer klinischen Norm ist nicht nur die Perfektion, sondern auch die Messung. Die Definition des Normalen geht dabei aus einem Set von Vergleichen hervor,

\section{KARGER}

Fax +497614520714

E-mail Information@Karger.de www.karger.com/journals/fkm

(C) 2001 S. Karger GmbH, Freiburg 
die entweder gegen eine dichotome Skala (normal vs. abnormal) oder innerhalb eines vorgeschriebenen Bereichs von Möglichkeiten (der statistischen Norm) durchgeführt werden. Dies führt auch dazu, dass in der Medizin eine zunehmende Abhängigkeit von Messwerten besteht, wenn zwischen normal und abnormal entschieden werden soll. Die Möglichkeit immer empfindlichere Messungen durchführen zu können, hat aber zu Folge, dass der Bereich dessen, was noch als normal anzusehen sei, immer kleiner und der Bereich des Abnormalen proportional dazu immer grösser wird.

Wird die Auffassung vertreten, dass normal auch perfekt sei, so darf nicht übersehen werden, dass auch der Begriff «perfekt» willkürlich definiert ist.

Was Perfektion bedeutet, unterliegt unter anderem Zeiteinflüssen, der persönlichen Einstellung oder dem Lebensalter. Der Verbrauch von Ressourcen in den industrialisierten Ländern auf der Jagd nach der perfekten Gesundheit ist gewaltig, obwohl der Gesundheitszustand der Bevölkerung weit davon entfernt ist, ideal zu sein. Die Bemühungen nach dem Erreichen eines optimalen Gesundheitszustands, die manchmal sogar das Ausmass einer Besessenheit annehmen, führen zu ständig steigenden Ausgaben, ohne dass das angestrebte Ziel wirklich erreicht wird. Zu bedenken ist aber auch, dass Perfektion, biologisch gesehen, nicht unbedingt günstig ist. Variabilität ist ein wichtiger biologischer Vorteil für das Überleben einer Spezies, und jede Uniformität vermindert die Anpassungsfähigkeit. Es ist wohl auch anmassend, wenn der Anspruch erhoben wird zu wissen, was Perfektion ausmache und dass man in der Lage sei, den Organismus so zu manipulieren, dass er Perfektion erreicht. Dies würde auch voraussetzen, dass man in der Lage sei, alle unvorherzusehenden Effekte zu kontrollieren.

Der Vorstellung von «normal» als «perfekt» entspricht auch die Auffassung des Organismus als Maschine, deren Teile ausgewechselt werden können, wenn sie nicht mehr optimal funktionieren. Das Streben nach Perfektion kann aber auch dazu führen, dass Gesundheit vordergründig nach dem äusseren Aussehen beurteilt wird. Auf dieser gedanklichen Basis erfolgt dann die Entwicklung von Produkten wie Shampoos, Supplementen, Kosmetika usw., die über eine «gesunde Haut», «gesundes Haar» oder «gesunde Nägel» usw. Gesundheit signalisieren sollen. Wird die perfekte Gesundheit nicht erreicht, so wird dies auch als persönliches Versagen dargestellt und empfunden. Hinter einer solchen Auffassung steht die Meinung, dass die perfekte Gesundheit durchaus erreichbar wäre, würde man nur mehr danach streben, sich gesünder ernähren und mehr körperliche Aktivität entfalten sowie die richtigen Produkte verwenden und sich besser erholen.

Die Gleichsetzung von «normal», «perfekt» und «gesund» führt möglicherweise auch dazu, dass Menschen mit einer gesundheitlichen Beeinträchtigung in der Gesellschaft auf jeden Fall als krank angesehen werden, unabhängig davon, ob sie sich persönlich eigentlich als gesund empfinden.
Der Drang nach perfekter Gesundheit hat auch Auswirkungen auf die Präventivmedizin. Bei der Krankheitsfrüherkennung treten zwei Probleme auf. Das intensive Bemühen, subklinische Anzeichen von Krankheiten zu entdecken, hat zur Folge, dass Messwerte erhoben werden ohne Rücksicht darauf, ob sich wirklich eine manifeste Krankheit entwickelt. Bei einer nicht unbeträchtlichen Zahl von Menschen werden so genannte Risikofaktoren gefunden, ohne dass in der Folge eine Krankheit auftritt, und umgekehrt treten manifeste Erkrankungen auch ohne vorher vorhandene Risikofaktoren auf. Das zweite Problem der Früherkennung ist ein ökonomisches. Es ist ein verständliches Ziel der Medizin, Krankheiten $\mathrm{zu}$ finden und zu behandeln. Dabei darf aber nicht vergessen werden, dass für Massnahmen der Frühdiagnostik erhebliche Geldmittel ausgegeben werden und eine Berechnung der Wirtschaftlichkeit dafür nicht existiert.

Bei allem Streben nach der perfekten Gesundheit muss zur Kenntnis genommen werden, dass dieses Streben an natürliche Grenzen stösst. Ein Beispiel für dieses Streben stellt die Gesundheitsdefinition der WHO dar, die allerdings eher als Ideologie aufzufassen ist und die mit der medizinischen Praxis nur wenig zu tun hat. Statt solcher idealisierender Gesundheitsdefinitionen wäre ein praxisbezogener Zugang dazu, was eine gute Gesundheit ausmacht, wichtiger. Eine solche Definition sollte auch die Variabilität als normal akzeptieren und sie nicht als Abweichung von der Perfektion auffassen. Dabei sollten auch die unabänderlichen Veränderungen des psychischen und psychischen Zustands von Menschen über die Zeit Berücksichtigung finden. Es sollte also eher der Prozess und nicht so sehr das Produkt als wesentliches Merkmal der Gesundheit im Vordergrund stehen.

Gesundheit wird immer in Relation zu bestimmten gesellschaftsbezogenen Annahmen definiert werden. Eine dieser Annahmen ist der Glaube an die Validität wissenschaftlicher Messungen. Genauso wichtig wäre aber auch eine Anerkennung der Variabilität als wichtiger Vorteil der lebenden Organismen. Die Auffassung der Medizin von dem was «normal» ist, sollte genügend umfassend sein, um auch die Variabilität und sogar die Schwächen der gesundheitlichen Verfassung des Menschen mit einschliessen zu können. Wir sollten selbstkritisch genug sein um zu erkennen, dass unsere Vorstellung von Perfektion notwendigerweise subjektiv, veränderbar und täuschend ist. Letztlich sollten wir zur Kenntnis nehmen, dass auch Krankheit ein normaler Teil des menschlichen Lebens ist.

W. Marktl, Wien

\section{Literatur}

Davies PV, Bradley JG: The meaning of normal. Perspect Biol Med 1996;40: 68-77. 